\title{
Meta-analysis of the incidence, prevalence and predictors of atrial fibrillation in rheumatic heart disease
}

\author{
Jean Jacques Noubiap, Ulrich Flore Nyaga, Aude Laetitia Ndoadoumgue, \\ Jan René Nkeck, Anderson Ngouo, Jean Joel Bigna
}

\section{APPENDIX}

\section{Supplementary Tables}

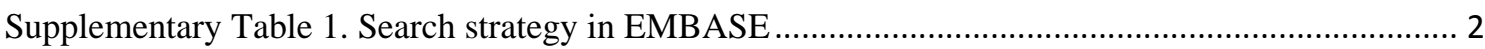

Supplementary Table 2. Summarized study characteristics ................................................................ 2

Supplementary Table 3. Individual characteristics of included studies ................................................ 3

Supplementary Table 4. MOOSE checklist................................................................................ 7

\section{Supplementary Figures}

Supplementary Figure 1. Studies selection

Supplementary Figure 2. Leave-one-out sensitivity analysis of the global prevalence of atrial fibrillation in rheumatic heart disease

Supplementary Figure 4. Meta-analysis of the prevalence of atrial fibrillation in patients in rheumatic heart disease who had valvular interventions

Supplementary Figure 5. Comparison of proportion of female sex between patients with and without atrial fibrillation in RHD

Supplementary Figure 6. Comparison N-terminal pro b-type natriuretic peptide concentration between patients with and without atrial fibrillation in RHD

Supplementary Figure 7. Comparison of mean diastolic pulmonary arterial pressure $(\mathrm{mmHg})$ between patients with and without atrial fibrillation in RHD

Supplementary Figure 8. Comparison of left ventricle end-systolic diameter between patients with and without atrial fibrillation in RHD

Supplementary Figure 9. Comparison of left ventricle end-diastolic diameter between patients with and without atrial fibrillation in RHD

Supplementary Figure 10. Comparison of mitral valve area $\left(\mathrm{cm}^{2}\right)$ between patients with and without atrial fibrillation in RHD 
Supplementary Table 1. Search strategy in EMBASE

\begin{tabular}{|l|l|}
\hline Search & Search terms \\
\hline$\# 1$ & $\begin{array}{l}\text { 'rheumatic heart disease'/exp OR 'rheumatic heart } \\
\text { disease' OR 'rhd' OR 'rheumatic mitral' OR 'rheumatic aortic' OR 'rheumatic } \\
\text { tricuspid' OR 'rheumatic pulmonary' OR 'bouillaud disease' OR 'bouillauds } \\
\text { disease' }\end{array}$ \\
\hline$\# 2$ & $\begin{array}{l}\text { 'atrial fibrillation'/exp OR 'atrial fibrillation' OR 'auricular fibrillation'/exp } \\
\text { OR 'auricular fibrillation' }\end{array}$ \\
\hline$\# 3$ & $\# 1$ AND \#2 \\
\hline
\end{tabular}

